\title{
GAUGE INVARIANCE OF THE DIRAC EQUATION
}

P. SMRZ

(Received 20 October 1971)

Communicated by C.A. Hurst

\section{Introduction}

The Dirac equation in its most usual form can be derived by the minimum action principle from the Lagrangian density

(1) $L(x)=\frac{i}{2}\left[\psi^{+}(x) \gamma^{4} \sum_{k=1}^{4} \gamma^{k} \partial_{k} \psi(x)-\left(\sum_{k=1}^{4} \partial_{k} \psi^{+}(x) \gamma^{4} \gamma^{k} \psi(x)\right)\right]-m \psi^{+}(x) \gamma^{4} \psi(x)$, where $\gamma^{k}(k=1,2,3,4)$ are the Dirac $4 \times 4$ matrices satisfying relations;

$$
\gamma^{i} \gamma^{k}+\gamma^{k} \gamma^{i}=2 g^{i k} I \text {, }
$$

$$
\begin{aligned}
& g^{i k}=0 \text { if } k \neq i, g^{44}=-g^{11}=-g^{22}=-g^{33}=1, \\
& \gamma^{4+}=\gamma^{4}, \gamma^{k+}=-\gamma^{k}(k=1,2,3) .
\end{aligned}
$$

$\psi(x)$ is a column matrix with 4 complex elements being functions of $x^{1}, x^{2}$, $x^{3}, x^{4}$, coordinates of the pseudo-Euclidean space with metric $g^{i k}$.

There are 16 independent matrices that can be written as products of the Dirac matrices. These matrices form a representation of the Lie algebra of $U(2,2)$ group; i.e., the group of linear transformations of a four-dimensional complex vector space which leaves quadratic form $\psi_{1}{ }^{*} \psi_{1}+\psi_{2}{ }^{*} \psi_{2}-\psi_{3}{ }^{*} \psi_{3}-\psi_{4}{ }^{*} \psi_{4}$ invariant. This fact is a rather unexplored aspect of the Dirac theory. A new approach to the theory can be formulated when $U(2,2)$ Lie algebra is taken as a starting point. Some interesting features of such an approach were already described in ref. [1]. The present paper puts the ideas on a firmer mathematical basis, and derives some consequences of the theory.

\section{Gauge transformation}

Let us consider a four-dimensional complex representation of $U(2,2)$ and a linear automorphism of the representation space defined simply by the action of $U(2,2)$ on $V$ :

$$
\tau_{g}: V \rightarrow V, \tau_{g}(\psi)=g \psi, \psi \in V, g \in U(2,2)
$$


Further define an automorphism of set $D=\left\{\gamma^{1}, \gamma^{2}, \gamma^{3}, \gamma^{4}\right\}$ of the Dirac matrices by

$$
\sigma_{g}: D \rightarrow D, \sigma_{g}(\Gamma)=g \Gamma \bar{g}^{-1}, \Gamma \in D, g \in U(2,2) .
$$

If $g$ is a fixed element of $U(2,2)$ and both $\tau_{g}$ and $\sigma_{g}$ are performed simultaneously, we shall call such a map a local gauge transformation under $U(2,2)$. As a map $\tau_{g}: V \rightarrow V$ it belongs to $U(2,2)$, and as a map $\sigma_{g}: D \rightarrow D$ it generates a Lie algebra automorphism of $\mathscr{U}$, the set of generators of $U(2,2)$. It also contains the usual quantum mechanical gauge transformation $\psi \rightarrow e^{i \alpha} \psi, \sigma_{y}(\Gamma)=\Gamma$, as $g$ is just $e^{i x} I$ in this case.

The local gauge transformation under $U(2,2)$ has one more pleasant property that will be described now. To be more specific, we choose a particular representation of the 16 elements of $\mathscr{U}$ :

$$
S_{k l}=\frac{1}{4}\left(\gamma^{k} \gamma^{l}+\gamma^{l} \gamma^{k}\right), \quad p_{k}=\frac{i}{2} \gamma^{k}, \quad q_{k}=\frac{1}{2} \gamma^{k} \gamma^{5},
$$

$\Lambda=\frac{i}{2} \gamma^{5}$, plus the matrix $i I$, where $I$ is the identity.

Matrix $\gamma^{5}$ is defined as

$$
\gamma^{5}=\gamma^{1} \gamma^{2} \gamma^{3} \gamma^{4}
$$

Commutation relations are then

$$
\begin{aligned}
& {\left[S_{k l}, S_{m n}\right]=g_{l m} S_{k n}+g_{k n} S_{l m}-g_{k m} S_{l n}-g_{l n} S_{k m}} \\
& {\left[S_{k l}, p_{m}\right]=g_{l m} p_{k}-g_{k m} p_{l}} \\
& {\left[S_{k l}, q_{m}\right]=g_{l m} q_{k}-g_{k m} q_{l}} \\
& \left.\left[p_{k}, q_{l}\right]\right\rceil=g_{k l} \Lambda \\
& {\left[\Lambda, p_{k}\right]=q_{k}} \\
& {\left[\Lambda, q_{k}\right]=p_{k}} \\
& {\left[\Lambda, S_{k l}\right]=0}
\end{aligned}
$$

Here $g_{k l}=g^{k l}(k, l=1,2,3,4)$ is the metric tensor defined in (2).

Matrices $S_{k l}$ generate the six-dimensional group of homogeneous Lorentz transformation $L \subset U(2,2)$, while $p_{k}$ and $q_{k}$ span subspaces $P$ and $Q$ respectively, with invariance properties with respect to the gauge transformation

$$
\sigma_{h}(P)=P, \quad \sigma_{h}(Q)=Q, \quad h \in L .
$$

This means that if we have a four-dimensional vector space $T$ with a basis $\left\{X_{1}, X_{2}, X_{3}, X_{4}\right\}$ we can define the action of $L$ on $T$ by considering a map 


$$
\mathscr{U} \otimes T \rightarrow \mathscr{U} \otimes T
$$

$$
\sum_{k=1}^{4} \sigma_{h}\left(p_{k}\right) X_{k}=\sum_{k=1}^{4} p_{k} h\left(X_{k}\right) .
$$

Such an action defines a Minkowski metric $(-,-,-,+)$ in $T$, as a symmetric bilinear form with this metric will be invariant with respect to the action of $L$ on $T$ defined by Eq. (8).

If we have a four-dimensional completely parallelisable manifold $M$, we can use (8) to define the action of $L$ on the tangent vector space $M_{p}$ at any point $p \in M$. In a coordinate system $\left(u^{1}, u^{2}, u^{3}, u^{4}\right)$ of $M$, basis vectors $K_{k}(u)$ at any point can be written as

$$
X_{k}(u)=\sum_{u=1}^{4} h_{k}^{\mu}(u) \frac{\partial}{\partial u^{\mu}} .
$$

Any set of continuous functions $h_{k}^{\mu}(u)$ satisfying $\operatorname{Det}\left(h_{k}^{\mu}(u)\right) \neq 0$ will then make $M$ the Riemann space of the general relativity with metric

$$
g^{\mu \nu}(u)=\sum_{k, l} h_{k}^{\mu}(u) h_{l}^{v}(u) g^{k l} .
$$

When the Dirac Lagrangian density (1) is rewritten using the notation introduced above as well as using coordinates $u^{\mu}(\mu=1,2,3,4)$ of $M$ instead of coordinates $x^{k}$, it looks like

where

$$
\begin{aligned}
L(u)= & \psi^{+}(u) \tilde{\Gamma} \sum_{k=1}^{4} p_{k} X_{k} \psi(u) \\
& +\sum_{k=1}^{4}\left(X_{k} \psi^{+}(u)\right) p_{k}^{+} \tilde{\Gamma} \psi(u)-m \psi^{+}(u) \tilde{\Gamma} \psi(u),
\end{aligned}
$$

$$
\tilde{\Gamma}=\left(\begin{array}{lll}
1 & & \\
& 1 & -1 \\
& &
\end{array}\right)=\gamma^{4}
$$

in the representation we are using. $L(u)$ is invariant with respect to the local gauge transformations as defined above. Moreover, it is seen from Eq. (8) that the local gauge transformations with respect to $L \subset U(2,2)$ is equivalent to the usual "Lorentz transformations of the Dirac equation" as it is treated in physics literature, where the Dirac matrices do not change, and $\psi \rightarrow h \psi, X_{k} \rightarrow h\left(X_{k}\right), h \in L$.

In this sense we can state that the invariance with respect to the local gauge transformations is more fundamental than the Lorentz invariance, as it can change a four-dimensional manifold into the physical space-time (defining a metric), while being made equivalent to the physical Lorentz transformations of the Dirac equation. 


\section{Global gauge invariance}

We shall now see how the Lagrangian (11) must be changed in order to make it invariant with respect to a global gauge transformation, defined in the same way as the local one, except that the group element $g$ is taken to be a differentiable function $g(u)$ on manifold $M$. A general prescription for such a procedure can be found in ref. [2] or [3]. $g(u)$ is expressed in terms of canonical coordinates of the group as

$$
g(u)=\exp \left(\sum_{a=1}^{16} \alpha^{a}(u) T_{a}\right)
$$

where $\alpha^{a}(\mu)$ where $\alpha(u)$ are differentiable functions of coordinates $u^{\mu}$, and $T_{a}$ are the matrices forming a basis of the Lie algebra of $U(2,2)$, as listed above (see (5)). Sixteen new vector valued functions $B_{\mu}^{a}(u)$ are introduced with a prescribed transformation properties with respect to the global transformation (up to the first order in $\alpha$ 's):

$$
B_{\mu}^{a}(u) \rightarrow B_{\mu}^{a}(u)+\sum_{b, c=1}^{16} \alpha^{c}(u) f_{c b}^{a} B_{\mu}^{b}(u)-\frac{\partial x^{a}(u)}{\partial u^{\mu}},
$$

where $f_{c b}^{a}$ are the structure coefficients of the Lie algebra of $U(2,2)$ when matrices $T_{a}$ are used as a basis. The derivative $\partial \psi / \partial u^{\mu}$ is replaced by a "covariant" derivative

$$
\psi_{\mu} \equiv \frac{\partial \psi}{\partial u^{\mu}}-\sum_{a=1}^{16} B_{\mu}^{a}(u) T_{a} \psi
$$

which transforms like

$$
\psi_{\mu}(u) \rightarrow g(u) \psi_{\mu}(u)
$$

The action of the global gauge transformations is then the same as that of the local ones, and the invariance of the Lagrangian is preserved. In this way terms containing the new fields $B_{\mu}^{a}(u)$ appear in the original Lagrangian, interpreted as the interaction terms, when a physical significance is given to them. The field corresponding to the matrix $i l$ in the algebra is, for example, interpreted as the electromagnetic field (compare eg. [4]). There will be further a group of six fields corresponding to $S_{k l}(k, l=1,2,3,4)$ (denoted by $B_{\mu}^{k l}=-B_{\mu}^{l k}$ ), a group of four fields corresponding to $p_{k}\left(B_{\mu}^{k(p)}\right)$, four fields corresponding to $q_{k}\left(B_{\mu}^{k(q)}\right.$, and finally one field corresponding to $\Lambda\left(B_{\mu}^{(\Lambda)}\right)$. If the invariance of the Lagrangian is to be preserved even in case the gauge transformations induced by $S_{k l}$ are interpreted as local Lorentz rotations according to relation (8), then $B_{\mu}^{k l}$ must transform as local tensors, while $B_{\mu}{ }^{k(p)}$ and $B_{\mu}^{k(q)}$ will transform as local vectors.

There is also a general form of a Lagrangian formed from the new fields, if the invariance with respect to transformation (13) is required. The lowest order invariant has a form [2] 


$$
L_{B}(u)=\sum_{a=1}^{16} \sum_{\mu, v} F_{\mu \nu}^{a} F_{a}^{\mu \nu},
$$

where

$$
F_{\mu \nu}^{a}=\frac{\partial B_{v}^{a}}{\partial u^{\mu}}-\frac{\partial B_{\mu}^{a}}{\partial u^{v}}-\sum_{b . c} f_{b c}^{a}\left(B_{\mu}^{b} B_{v}^{c}-B_{v}^{b} B_{\mu}^{c}\right)
$$

Index $a=1, \cdots, 16$ is lowered by using the Lie algebra metric $g_{a b}=\Sigma_{c, d} f_{a d}^{c} f_{b c}^{d}$ and indices $\mu, v=1, \cdots, 4$ are raised by using the Riemann metric $g^{\mu \nu}(u)$.

\section{A particular solution}

It was found that a solution satisfying the minimum action principle with the Lagrangian (12) of the new fields exists and can have a physical interpretation under some simplifying conditions.

Firstly, as we interpret that part of the gauge transformations which is induced by $S_{k l}$ in the same way as in ref. [2] (i.e., as local Lorentz rotations) we use the relation

$$
B_{\mu}^{i k}=\sum_{v=1}^{4} h^{k v} \frac{\partial h_{v}^{i}}{\partial u^{\mu}}-\sum_{\rho, v} h_{\rho}^{i} h^{k v} \Gamma_{\mu \nu}^{\rho}
$$

derived in ref. [2].

Further we put the electromagnetic field as well as field $B_{\mu}^{(\Lambda)}$ identically equal to zero. Finally we observe that the interaction term corresponding to the fields $B_{\mu}^{k(p)}$ and $B_{\mu}^{k(q)}$ in the Dirac Lagrangian has the form

$$
\psi^{+}(u) \tilde{\Gamma} \sum_{\mu, k} B_{\mu}^{k(p)} h_{k}^{\mu} \psi(u)=\phi(u) \psi^{+}(u) \tilde{\Gamma} \psi(u)
$$

which is a scalar function with respect to both the local Lorentz rotations and a general change of coordinates $u^{\mu}$. This suggests a possible origin of the mass term $m \psi^{+} \tilde{\Gamma} \psi$ as such an interaction term where $\phi(u)$ happens to be constant. Therefore, we attempt to find a solution with $\phi(u)=m$.

If we look only for a spherically symmetric static solution some further unknown functions are eliminated, and a system of two differential equations for two unknown functions

and

$$
\gamma(r)=g_{44}(r)=-\frac{1}{g_{33}(r)}
$$

$$
f(r)=\sum_{k, \mu} B_{\mu}^{k(q)} \cdot h_{k}^{\mu}
$$


is obtained. The simplification of the original system of Euler equations is straightforward but tedious, and we present only the final system:

$$
r^{2} \gamma^{\prime \prime \prime}+4 r \gamma^{\prime \prime \prime}-2 \gamma^{\prime \prime}-\frac{4}{r^{2}}(1-\gamma)+4 r^{2}\left[\left(f^{\prime}\right)^{2}+f f^{\prime \prime}\right]=0
$$

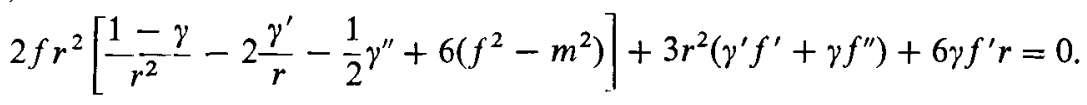

Even this simplified system is mathematically rather complicated, and a general discussion of possible solutions is difficult. Nevertheless, a specific solution is of an interest.

If $f(r)=a$ (constant) for all values of $r$, the equations are satisfied by

$$
\gamma(r)=1-\frac{2 k m}{r}-\left(m^{2}-a^{2}\right) r^{2} .
$$

This is the spherically symmetric solution of the Einstein equations with a "cosmological" term corresponding to the "radius of the universe" equal to $1 / \sqrt{m^{2}-a^{2}}$ (see [5]). When $a=m$ we obtain the Schwarzchild solution of the Einstein equations ( $k$ is then the Newton's gravitational constant). For $a \rightarrow 0$ the radius becomes $1 / \mathrm{m}$ which is usually considered as a reasonable estimate of the radius of an elementary particle with mass $m$.

\section{References}

[1] P. Smrz, 'Philosophy of a Space Generalization and the Problem of Spinors in General Relativity,' Il Nuovo Cim. 65B (1970), 147-157.

[2] R. Utiyama, 'Invariant Theoretical Interpretation of Interaction', Phys. Rev. 101 (1956), 1597-1607.

[3] T.W.B. Kibble, 'Lorentz Invariance and the Gravitational Field', J. Math. Phys. 2 (1961), 212-221.

[4] S.S. Schweber, An Introduction to Relativistic Quantum Field Theory (Row, Peterson \& Co., 1961), page 272.

[5] A.S. Eddington, The Mathematical Theory of Relativity (Cambridge Univ. Press, 1957). page 100 .

University of Newcastle

New South Wales, Australia 